\title{
Déclin des deux plus grands glaciers des Alpes françaises au cours du XX|e siècle : Argentière et Mer de Glace
}

\author{
Christian Vincent ${ }^{1}$, Vincent Peyaud ${ }^{1}$, Olivier Laarman ${ }^{1}$, Delphine Six ${ }^{1}$, \\ Adrien Gilbert ${ }^{2}$, Fabien Gillet-Chaulet ${ }^{1}$, Étienne Berthier ${ }^{3}$, \\ Samuel Morin ${ }^{4}$, Deborah Verfaillie ${ }^{4,5}$, Antoine Rabatel ${ }^{1}$, \\ Bruno Jourdain 1 , Jordi Bolibar ${ }^{1}$ \\ 1. Institut des géosciences de l'environnement, Université Grenoble Alpes / \\ CNRS, Grenoble \\ 2. Department of Geosciences, University of Oslo, Oslo, Norvège \\ 3. Laboratoire d'études en géophysique et Océanographie spatiales, \\ Université de Toulouse / Cnes / CNRS, Toulouse \\ 4. Université Grenoble Alpes, Météo-France, Centre d'études de la neige, \\ Grenoble \\ 5. Barcelona Supercomputing Center, Barcelone, Espagne
}

\section{Résumé}

Des modélisations ont été réalisées sur les deux plus grands glaciers des Alpes françaises afin d'estimer leur évolution au cours du XXI' ${ }^{\mathrm{e}}$ siècle. Pour un scénario climatique intermédiaire avec réduction des émissions de gaz à effet de serre avant la fin du XXI ${ }^{\mathrm{e}}$ siècle (RCP 4.5), les simulations indiquent que le glacier d'Argentière devrait disparaître vers la fin du $\mathrm{XXI}^{\mathrm{e}}$ siècle et que la surface de la Mer de Glace pourrait diminuer de $80 \%$. Dans l'hypothèse la plus pessimiste d'une croissance ininterrompue des émissions de gaz à effet de serre (RCP 8.5), la Mer de Glace pourrait disparaître avant 2100 et le glacier d'Argentière une vingtaine d'années plus tôt. es glaciers méritent une attention particulière en climatologie, car ce sont des indicateurs remarquables des changements climatiques (Oerlemans, 2001 ; Thibert et al., 2018 ; Huss et al., 2017 ; Zemp et al., 2019). Le devenir des glaciers et la conséquence de leur évolution sur l'hydrologie, les infrastructures touristiques et les risques d'origine glaciaire sont aussi des questions cruciales pour les habitants des vallées alpines (Kaser et al., 2010 ; Huss, 2011 ; Huss et Hock, 2018). Au cours des trois dernières décennies, les glaciers alpins ont vu leur masse

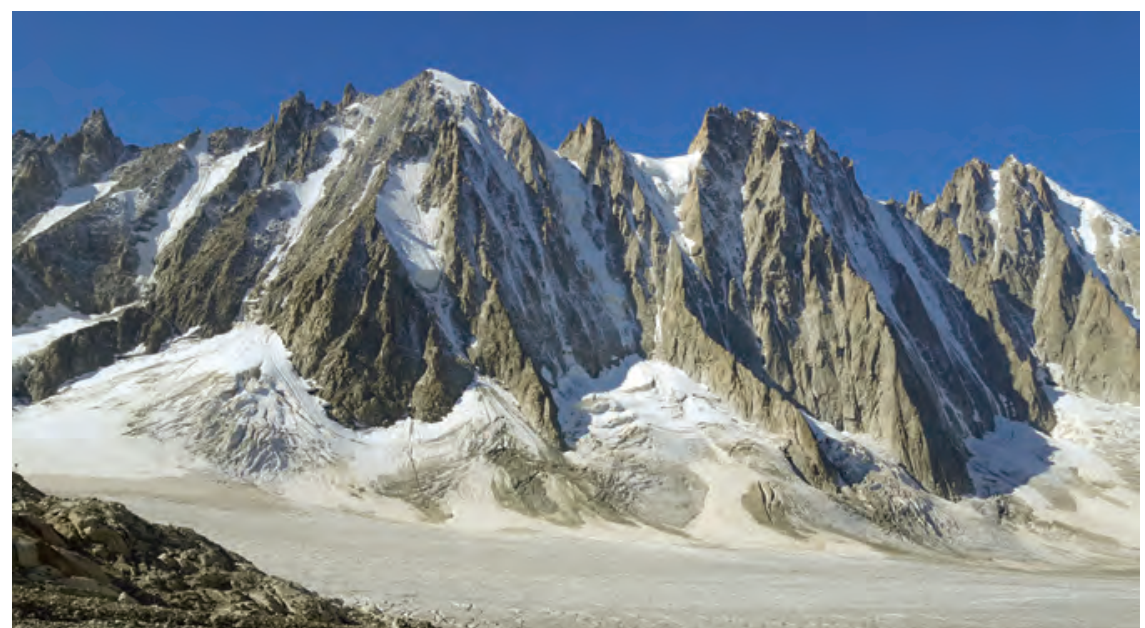

Figure 1. Glacier d'Argentière. Photo : C. Vincent, septembre 2018. diminuer d'une façon totalement inédite par rapport à leurs fluctuations connues au cours des 150 dernières années (WGMS, 2015 ; Zemp et al., 2013, 2015 ; Fischer et al., 2015). En outre, la fonte s'est nettement accentuée depuis 2002 (Vincent et al., 2017 ; Huss et al., 2017). Plusieurs études indiquent que les glaciers dont la zone d'accumulation n'excède pas $3500 \mathrm{~m}$ dans les Alpes risquent de disparaître avant la fin de ce siècle (Le Meur et al., 2007 ; Jouvet et al., 2011). La prévision de l'évolution des glaciers pour le siècle à venir nécessite de connaître la répartition actuelle des 


\section{Abstract}

Decay of the two largest glaciers in the French Alps during the 21st century : Argentière and Mer de Glace

Numerical simulations have been performed to infer the fate of the two largest glaciers of the French Alps during the $21^{\text {st }}$ century. For the scenario with reduction of greenhouse gas emissions before the end of the $21^{\text {st }}$ century (RCP 4.5), the model shows that the Argentière glacier might disappear towards the end of the $21^{\text {st }}$ century and the Mer de Glace surface area could decrease by $80 \%$. For the most pessimistic scenario with a continuous increase in greenhouse gas emissions (RCP 8.5), Argentière and Mer de Glace might disappear before 2080 and before the end of the 21st century, respectively.

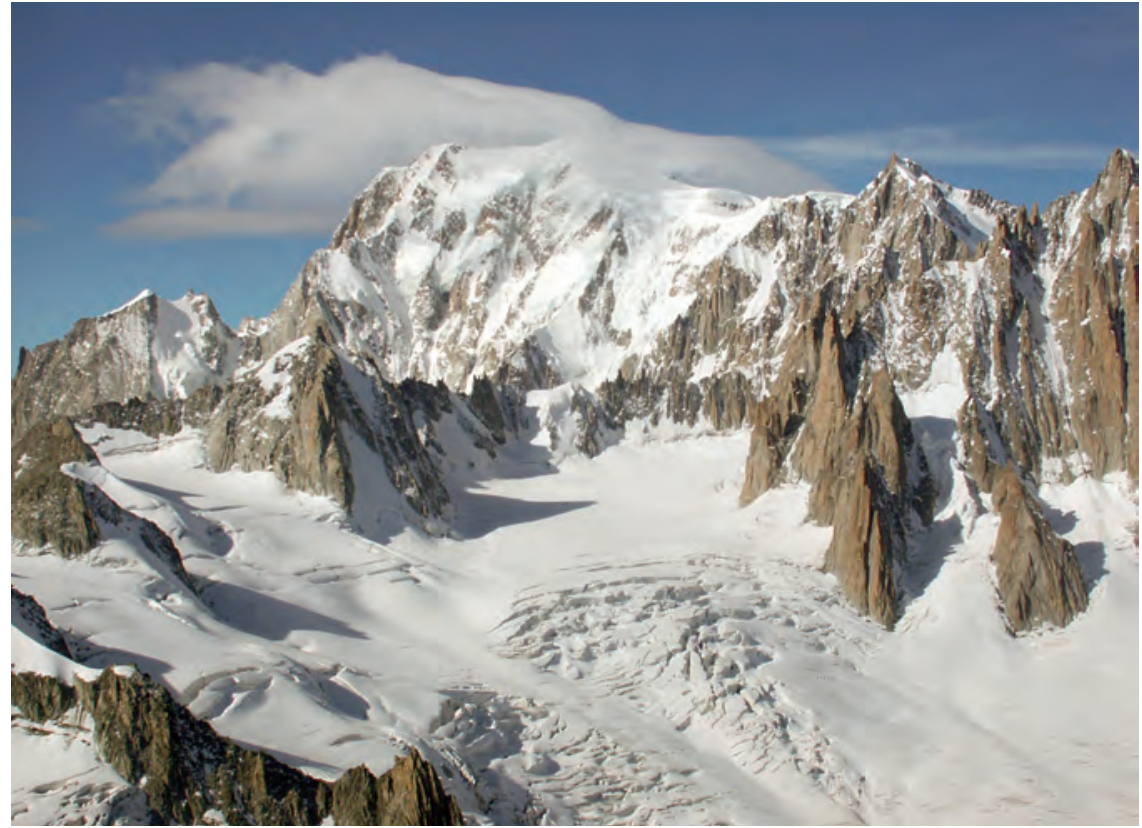

Figure 2. Bassin d'accumulation de la Mer de Glace. En arrière-plan, le sommet du mont Blanc (sous les nuages). Photo : C. Vincent, 2004.

épaisseurs de glace, mais aussi l'évolution du climat et des variables météorologiques locales qui gouvernent les échanges d'énergie et de masse. Enfin, il est essentiel d'intégrer à la modélisation l'écoulement du glacier qui redistribue la glace des zones d'accumulation aux zones d'ablation.

La présente étude a pour objectif de reconstituer l'évolution passée et de déterminer l'évolution possible, au cours du XXI ${ }^{\mathrm{e}}$ siècle, de deux grands glaciers du massif du Mont-Blanc que sont les glaciers d'Argentière et de la Mer de Glace (figures 1 et 2), en utilisant différents scénarios et modèles climatiques.

\section{Les variations passées des glaciers d'Argentière et de la Mer de Glace}

Les glaciers d'Argentière et de la Mer de Glace ont fait l'objet d'observations glaciologiques de longue date. Les fronts et les variations d'épaisseur des langues glaciaires sur quelques sections transversales ont été mesurés par Joseph Vallot à la fin du XIX ${ }^{\mathrm{e}}$ siècle, puis par des agents des Eaux et Forêts au début du $\mathrm{XX}^{\mathrm{e}}$ siècle (figure 3). Les bilans de masse ${ }^{1}$ de surface des langues glaciaires sont mesurés directement respectivement depuis 1976 et 1979 pour les glaciers d'Argentière et de la Mer de Glace. Ces observations ont été étendues en zone d'accumulation et à l'échelle hivernale et estivale à partir de 1994 (Vincent, 2002) et intégrées dans le service national d'observations Glacioclim $^{2}$ en 2002. En outre, la carte du massif du Mont-Blanc de J. Vallot (Vallot, 1905), les restitutions photogrammétriques des clichés aériens anciens (Vincent, 2002 ; Vincent et al., 2009, 2014) et les observations satellitaires récentes (Berthier et Vincent, 2012 ; Berthier et al., 2014) ont permis de calculer des bilans géodésiques (variations de volume) couvrant plusieurs décennies.

La figure 4 indique que, depuis le début $\mathrm{du} \mathrm{XX}^{\mathrm{e}}$ siècle, les glaciers d'Argentière et de la Mer de Glace ont perdu respectivement 34 et 45 mètres équivalent en eau, soit 38 et $50 \mathrm{~m}$ d'épaisseur de glace, en moyenne sur toute leur surface. Ces pertes représentent respectivement 25 et $32 \%$ de leurs épaisseurs moyennes depuis le début du XXe siècle. Cette figure montre aussi que la décroissance n'est pas linéaire dans le temps et l'on note très clairement la petite crue glaciaire (période de gain de masse) entre 1955 et le milieu des années 1980. Les variations d'altitude de la surface de quelques sections transversales

1. Le bilan de masse de surface est la différence entre l'accumulation et l'ablation. Le bilan de masse ponctuel annuel est mesuré par carottage en zone d'accumulation et à l'aide de balises d'ablation dans la zone d'ablation. Le bilan de masse cumulé est la somme des bilans annuels, 2. https://glacioclim.osug.fr intégrés sur l'ensemble de la surface du glacier. 


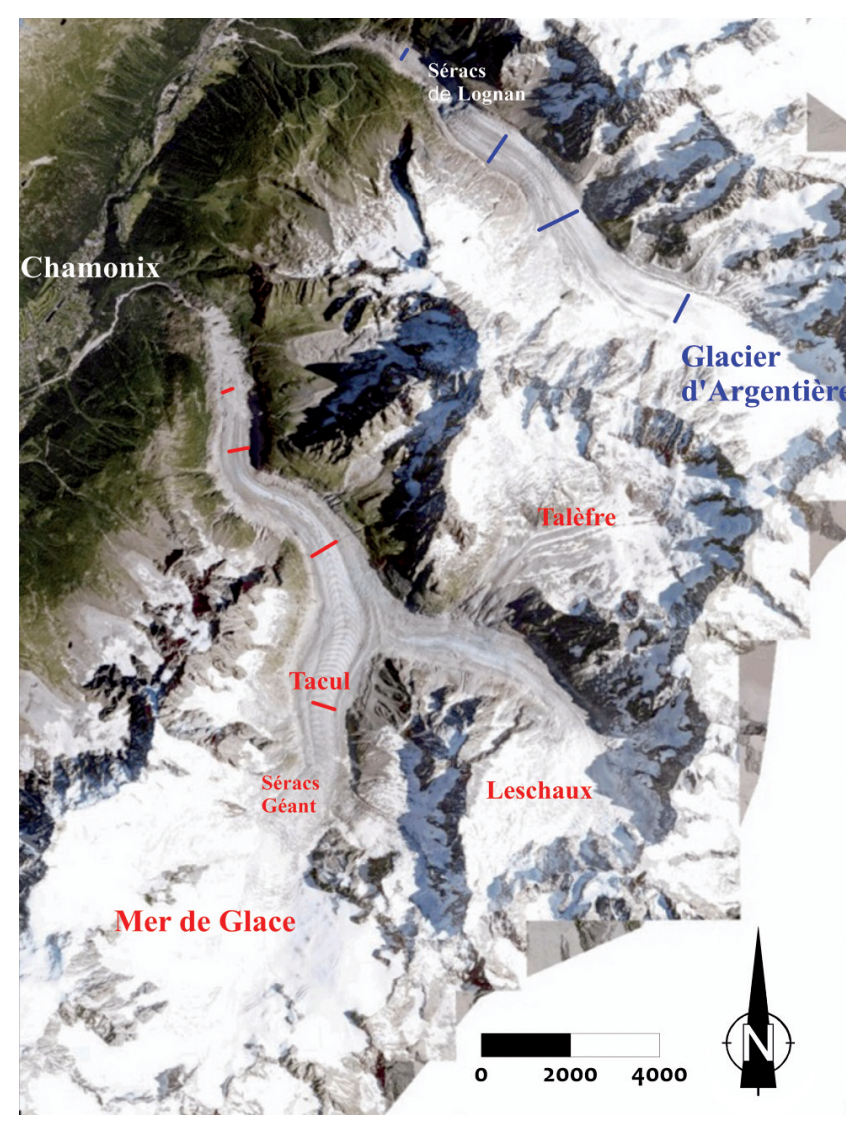

Figure 3. Carte des glaciers d'Argentière et de la Mer de Glace. Les lignes représentent les sections transversales mesurées depuis la fin du XIXe siècle (figure 5) (profils 7, 5, 4 et 2 du haut en bas pour le glacier d'Argentière et profils Tacul, Trélaporte, Echelets et Montenvers pour la Mer de Glace). L'arrière-plan est une orthophoto-aérienne de I'Institut national géographique datant de 2015 (https://www.geoportail.gouv.fr/).

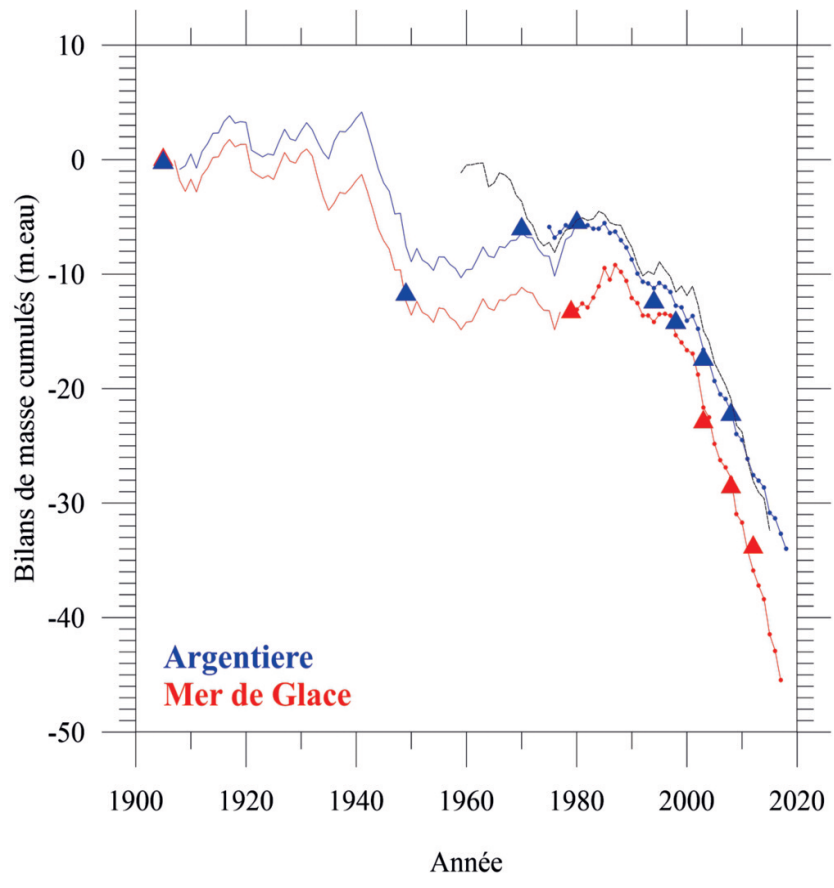

Figure 4. Bilans de masse de surface cumulés (en mètres d'eau) des glaciers d'Argentière (en bleu) et de la Mer de Glace (en rouge) entre 1905 et 2018. Les triangles correspondent aux mesures géodésiques (photogrammétrie aérienne ou mesures satellitaires). Les points résultent des observations directes de terrain. Les lignes bleue et rouge représentent une reconstitution des bilans de masse à partir de variables météorologiques (Vincent et al., 2009). La courbe noire résulte du modèle degré-jour utilisé dans cette étude à partir des réanalyses Safran pour le glacier d'Argentière.

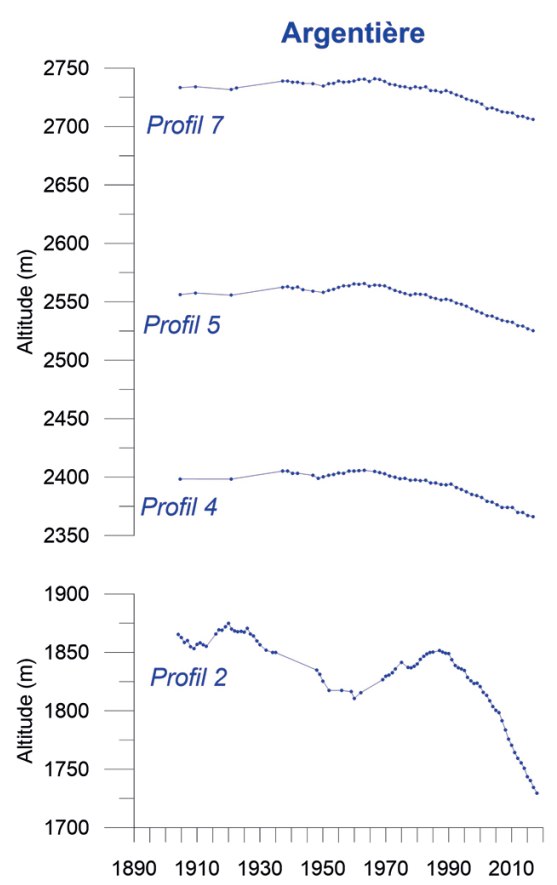

Figure 5. Variations d'altitude (en mètre) de la surface des sections transversales des langues glaciaires d'Argentière (gauche) et de la Mer de Glace (droite) depuis la fin du XIXe siècle. Voir la figure 3 pour la localisation des sections transversales.

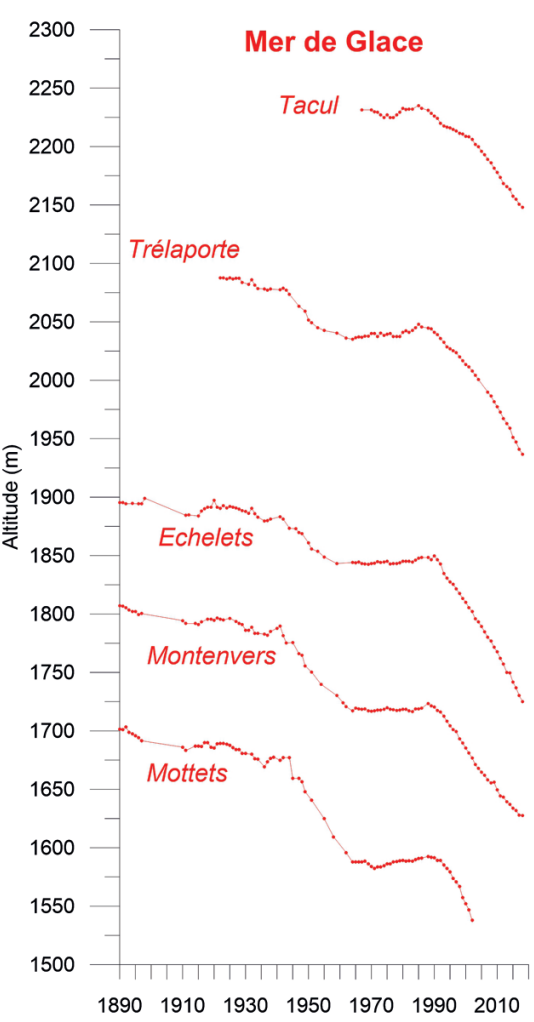

\section{Des modèles qui} permettent de calculer les changements d'épaisseur et de longueur

Plusieurs étapes sont nécessaires pour modéliser l'évolution passée et future des glaciers. Dans un premier temps, il est nécessaire de calculer le bilan de masse de surface à partir des variables météorologiques. Des modèles de complexité variable peuvent être utilisés. Gabbi et al. (2014) et Réveillet et al. (2018) ont montré que les modèles de type « degré-jour », qui calculent la 
fonte à partir de la température de l'air, sont adaptés pour simuler la fonte pour de longues périodes de temps. La principale source d'incertitude réside dans l'accumulation de neige hivernale qui conditionne l'albédo (pouvoir réfléchissant) de la surface et donc la fonte lors du printemps et l'été qui suivent (Réveillet et al., 2018). Dans cette approche " degré-jour » que nous utilisons pour cette étude, la fonte s'exprime simplement comme une fonction de la température de l'air (quand elle est supérieure à $0{ }^{\circ} \mathrm{C}$ ) en utilisant des facteurs respectifs de 4 et $6 \mathrm{~mm}$ équivalent en eau de fonte par degré positif journalier pour la neige et la glace (Vincent, 2002). La détermination de l'accumulation de la neige est beaucoup plus problématique, car elle dépend non seulement des précipitations solides, mais également de la redistribution de la neige par le vent ou des avalanches. En ce qui concerne les glaciers d'Argentière et de la Mer de Glace, les bilans de masse de surface hivernaux et estivaux sont estimés à partir de mesures depuis 1994 en une cinquantaine de points, ce qui permet de déterminer une relation empirique entre l'accumulation mesurée sur le glacier et la précipitation solide (neige) mesurée indépendamment en dehors du glacier pour des années très contrastées. En outre, les bilans de masse de surface de l'ensemble du glacier (moyenne sur tout le glacier) sont bien connus depuis le milieu du $\mathrm{XX}^{\mathrm{e}}$ siècle grâce aux mesures géodésiques. Ainsi, les bilans de masse annuels et la fonte calculée à l'aide d'un modèle degré-jour appliqué sur plusieurs décennies permettent d'ajuster les paramètres d'accumulation. Pour cela, on soustrait la fonte calculée du bilan annuel mesuré pour en déduire l'accumulation moyenne sur l'ensemble du glacier. Les bilans de masse de surface sont ainsi calculés en chaque point du glacier et pour chaque jour. Intégrés sur toute la surface, ces bilans de masse de surface permettent de calculer un bilan de masse d'ensemble du glacier ou une variation de volume. Pour cette étude, nous avons utilisé la réanalyse Safran (Durand et al., 2009) disponible pour la période de 1958 à 2018, fournissant les valeurs quotidiennes de précipitation et de température par niveaux d'altitudes de $300 \mathrm{~m}$ dans le massif du Mont-Blanc.

À partir de ces résultats, les variations d'épaisseur en chaque point du glacier, qui dépendent à la fois du bilan de masse de surface et de l'écoulement du glacier, peuvent être calculées avec des approches de complexité variable. Les modèles 3D d'écoulement de glace, par exemple Elmer/Ice (Gagliardini et al., 2013), sont des outils puissants pour représenter la dynamique du glacier (résolution du champ de vitesses d'écoulement), mais ils nécessitent une bonne connaissance de la topographie sous-glaciaire et sont particulièrement sensibles à la loi de frottement de la base du glacier sur le lit rocheux (Brondex et al., 2017) qui reste une source d'incertitude majeure. Nous avons opté, dans le cadre de la présente étude, pour un modèle dit " paramétré » qui permet de convertir le bilan de masse d'ensemble $\mathrm{du}$ glacier en variations d'épaisseur. Cette approche permet d'assurer la conservation de la masse sans représenter explicitement la dynamique, c'est-à-dire le champ de vitesse du glacier. En revanche, elle nécessite beaucoup d'observations de variations d'épaisseur dans le passé. L'approche est donc très adaptée pour des glaciers qui sont suivis depuis plusieurs décennies, comme c'est le cas pour la Mer de Glace et le glacier
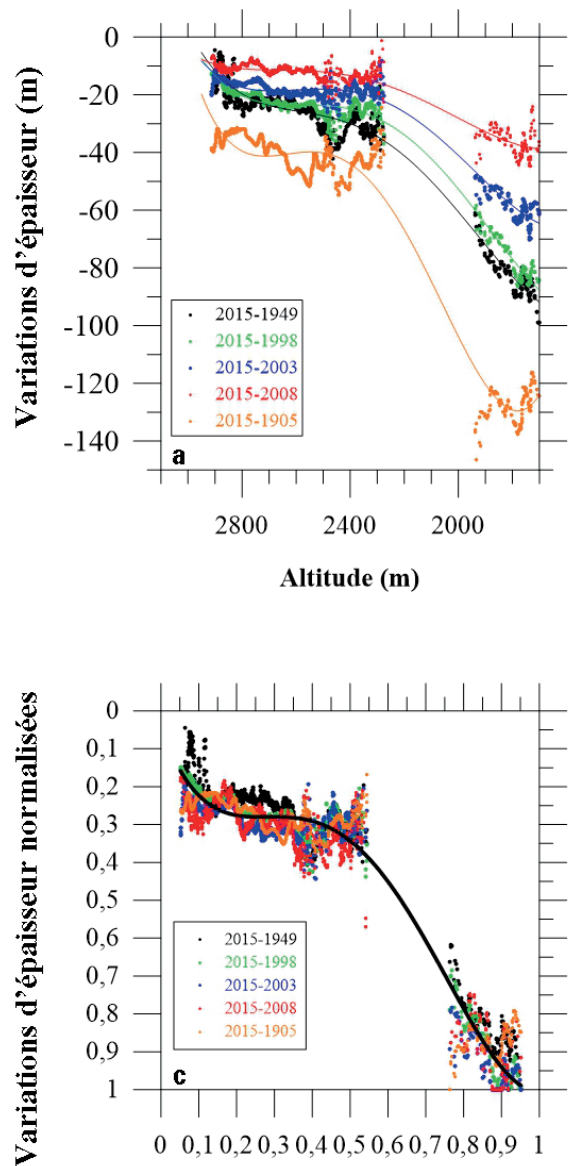

Altitude normalisée

Figure 6. Variations d'épaisseur (en $\mathrm{m}$ ) des glaciers (a) d'Argentière et (b) de la Mer de Glace en fonction de l'altitude, pour différentes périodes. Variations d'épaisseur normalisées sur ces mêmes glaciers (c et d).
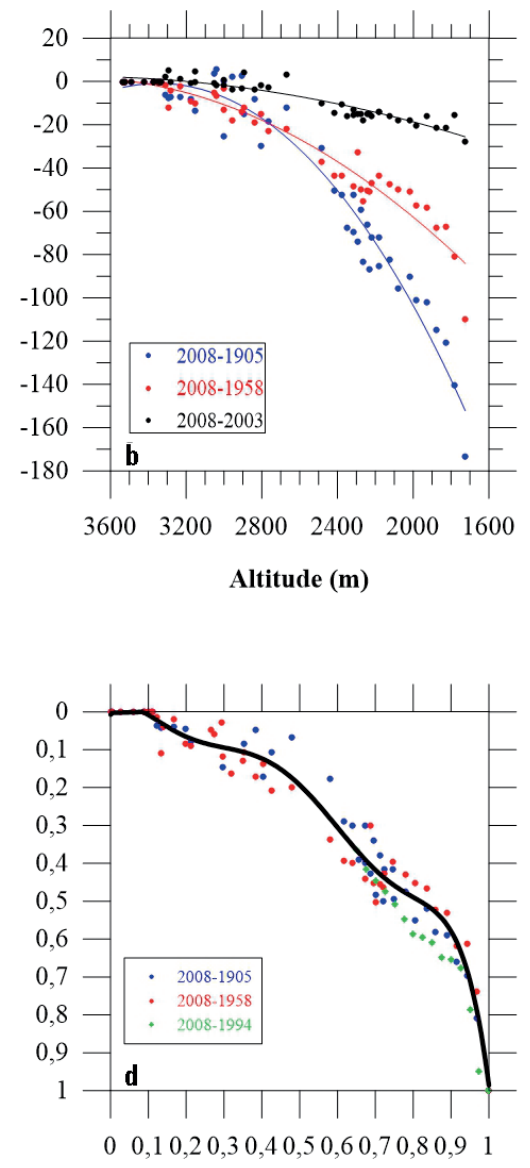

Altitude normalisée

d'Argentière. La méthode ne nous affranchit pas de la connaissance du lit rocheux, mais elle y est beaucoup moins sensible, car les fluctuations d'épaisseur du glacier sont insensibles et au frottement du glacier sur son lit. La méthode est décrite en détail dans Huss et al. (2010) et Vincent et al. (2014). Les variations d'épaisseur observées dans le passé (figures $6 a$ et 6b), sur plusieurs années ou plusieurs décennies, sont normalisées, c'est-àdire divisées par la perte d'épaisseur maximum, et les dénivelées sont divisées par la dénivelée totale du glacier. Les périodes pour établir ces relations ont été sélectionnées en fonction des données disponibles. Ces fonctions normalisées (figures $6 \mathrm{c}$ et $6 \mathrm{~d}$ ), relativement constantes dans le temps pour chaque glacier considéré, peuvent être utilisées dans le futur moyennant la connaissance du bilan de masse et en faisant l'hypothèse de stationnarité de ces relations. Lors de l'application de cette approche paramétrée pour les projections, la variation d'épaisseur en chaque point à la pente, à l'hydrologie sous-glaciaire 
du glacier est ainsi calculée en fonction du bilan de masse de surface de l'ensemble du glacier, en utilisant cette fonction normalisée. Grâce aux variations d'épaisseurs obtenues, les nouvelles altitudes de la surface glaciaire sont calculées chaque année. Cette itération annuelle des altitudes permet de tenir compte de la rétroaction de la baisse de l'altitude du glacier sur les bilans de masse de surface. Lorsque le lit rocheux est atteint, la surface est déglacée. La position du front et une nouvelle surface du glacier sont calculées pour l'année suivante.

\section{Topographie du lit rocheux de chacun des glaciers}

La connaissance de la topographie du socle rocheux est essentielle pour simuler l'évolution des glaciers dans le futur. Elle n'a été mesurée que pour quelques glaciers dans le monde. Pour les autres glaciers, la topographie du socle rocheux est estimée à partir de modèles plus ou moins complexes comportant de grandes incertitudes (Farinotti et al., 2017).

Sur les glaciers d'Argentière et de la Mer de Glace, beaucoup d'investigations géophysiques ont été réalisées avec des méthodes sismiques ou par forages dans les années 1960 et 1970 pour déterminer le lit rocheux des langues glaciaires, principalement à des fins hydroélectriques (Vincent et al., 2009 ; Vincent et Moreau, 2016). Le lit rocheux de la partie haute du glacier d'Argentière a été étudié beaucoup plus récemment par méthodes radar (Rabatel et al., 2018) comme celui de la Mer de Glace (données non publiées). Les méthodes d'inversion qui utilisent un modèle d'écoulement et les flux de glace (Gilbert et al., 2018) ont permis de compléter la connaissance des épaisseurs de glace dans la partie haute du glacier d'Argentière, en particulier sur les affluents (glaciers des Rognons, des Améthystes et du Tour Noir) dépourvus de mesures d'épaisseur. Le lit rocheux de la partie supérieure de la Mer de Glace est beaucoup plus problématique, car il n'est connu que pour sept sections transversales dans cette zone, ce qui représente une petite partie de la zone d'accumulation. Nous avons comblé ces lacunes en utilisant la base de données de Huss et Farinotti (2012) qui ont utilisé une méthode basée sur un calcul de flux de glace pour estimer les lits rocheux de différents glaciers.

\section{Les scénarios et modèles climatiques utilisés}

Les données de précipitations et de températures journalières utilisées pour nos simulations d'évolution future sont issues des résultats de modèles de climat régionaux, contraints par les modèles climatiques à l'échelle planétaire utilisés pour les travaux du Giec (voir encadré : «Les scénarios climatiques »). En l'occurrence, nos travaux se sont appuyés sur les projections de plusieurs couples de modèles globaux (GCM) et régionaux (RCM) produits dans le cadre du projet Euro-Cordex, dont les résultats ont été ajustés pour les rendre applicables en zones de montagne en utilisant la méthode Adamont (Verfaillie et al., 2017). Cette méthode exploite les données climatologiques issues de la réanalyse Safran (Durand et al., 2009) et caractérise les liens statistiques entre Safran et chaque modèle de climat pendant la période historique (1950-2005). Ces relations sont ensuite utilisées pour ajuster les projections futures 2006-2100, en utilisant plusieurs scénarios d'émission de gaz à effet de serre (RCP).

La figure 7 présente l'évolution des températures et des précipitations annuelles à $2700 \mathrm{~m}$ pour 29 réalisations de ce système de projection climatique, entre 1982 et 2100 . Ce sous-ensemble de projections climatiques (Verfaillie $e t$ al., 2018) couvre une bonne partie de la gamme de variation climatique pour la période d'intérêt. La figure 7 montre l'existence de fluctuations significatives de température et de précipitation, et une tendance nette pour les températures, particulièrement accentuée et différenciée selon les scénarios à partir de 2050. Pour le scénario RCP 8.5, l'accroissement de température sur l'ensemble de la période atteint 6 à $7{ }^{\circ} \mathrm{C}$, alors que pour le RCP 2.6 et RCP 4.5 les accroissements moyens sont respectivement de l'ordre de 2 et $3{ }^{\circ} \mathrm{C}$. Les valeurs de précipitation montrent surtout de fortes fluctuations avec une variabilité élevée aux échelles décennales et l'absence de tendance marquée sur l'ensemble de la période.

\section{Simulations des glaciers d'Argentière et de la Mer de Glace jusqu'en 2100}

Les évolutions des glaciers d'Argentière et de la Mer de Glace sont calculées à partir des modèles numériques de terrain disponibles les plus récents, respectivement en 2015 et 2008, en utilisant les données de précipitations et températures journalières des modèles climatiques. Ces données permettent de calculer les bilans de masse de surface passés et futurs et l'altitude de la ligne

\section{Les scénarios climatiques}

Pour analyser les changements climatiques à attendre dans le futur, le groupe d'experts intergouvernemental sur l'évolution du climat (Giec) utilise quatre trajectoires d'émissions et de concentrations de gaz à effet de serre, d'ozone et d'aérosols. Ces scénarios sont appelés representative concentration pathways (RCP). Dans le scénario RCP 2.6, les émissions de gaz à effet de serre diminuent après 2030 et les concentrations diminuent après 2050. Dans le scénario RCP 4.5, les émissions de gaz à effet de serre diminuent après 2050 et les concentrations se stabilisent vers la fin du XXle siècle. Dans le scénario RCP 8.5 , les émissions de gaz à effet de serre se stabilisent avant la fin du XXle siècle mais les concentrations continuent d'augmenter au-delà du XXle siècle.

Ces scénarios sont traduits en termes de forçage radiatif. Le bilan radiatif représente la différence entre le rayonnement solaire reçu et le rayonnement infrarouge réémis par la planète, calculé au sommet de la troposphère. II est exprimé en $W / \mathrm{m}^{2}$ et correspond à une évolution du forçage radiatif d'ici 2100. Le scénario RCP 2.6, le plus optimiste, correspond à un forçage radiatif de $2,6 \mathrm{~W} / \mathrm{m}^{2}$ en 2100 , alors que le scénario RCP 8.5 , le plus pessimiste, correspond à un forçage radiatif de $8,5 \mathrm{~W} / \mathrm{m}^{2}$ en 2100 (http://www.ipcc.ch/). 

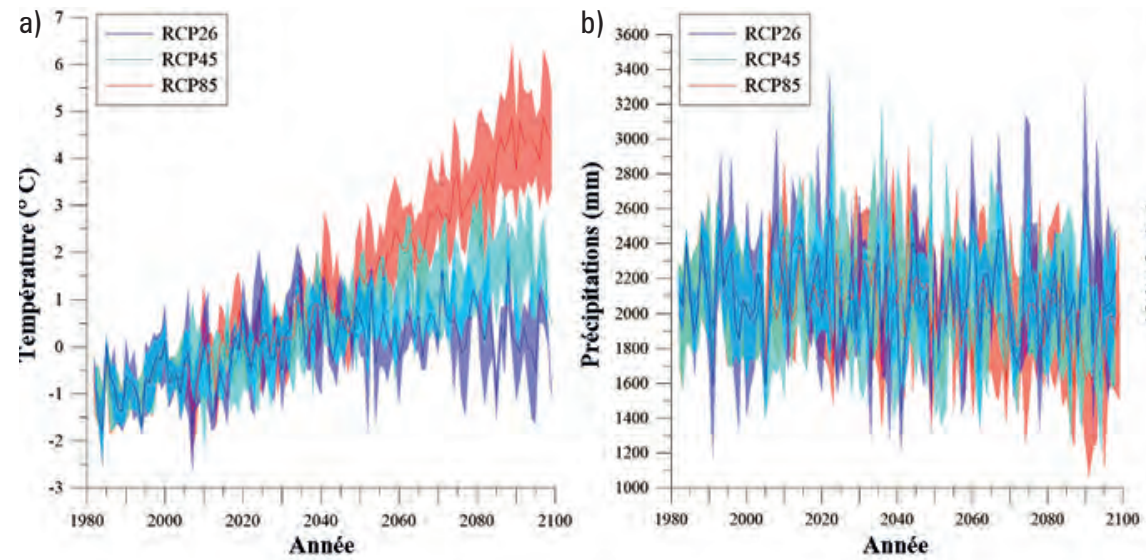

Figure 7. Évolution des (a) températures (en ${ }^{\circ} \mathrm{C}$ ) et (b) des précipitations (en mm/an) annuelles à $2700 \mathrm{~m}$ d'altitude pour les différents scénarios climatiques (moyenne et écart type des valeurs fournies pour chaque année par les couples GCM/RCM d'un RCP donné).

d'équilibre ${ }^{3}$ (figure 8 ). À la fin du $\mathrm{XXI}^{\mathrm{e}}$ siècle, les projections indiquent que l'altitude de la ligne d'équilibre pourrait se situer vers $3500 \mathrm{~m}$ suivant la moyenne des modèles du scénario RCP 8.5 ou vers $3000 \mathrm{~m}$ suivant le scénario RCP 2.6. Ces écarts révèlent des différences considérables de bilans de masse de surface d'environ 4 à $5 \mathrm{~m}$ d'eau par an entre ces scénarios. Les conséquences sont très importantes sur les simulations des changements d'épaisseur. Sur la figure 9, nous avons reporté les résultats de simulation d'un couple RCM/GCM CLMcom-CNRMCM5-RCP.45 (CC-4.5) pour le glacier d'Argentière et la Mer de Glace entre 2020 et 2100 . Bien que ce modèle climatique soit relativement «modéré » dans le sens où il se rapproche de la moyenne d'ensemble des différentes simulations disponibles pour le RCP 4.5 , on peut voir, à partir de la figure 9 , que les changements de volume et de surface seraient considérables.

Le glacier d'Argentière présente une rupture de pente, à la chute de séracs de Lognan, entre 2400 et $1900 \mathrm{~m}$ d'altitude. La langue du glacier d'Argentière est déconnectée de la partie haute depuis 2011. La partie basse est donc constituée de glace régénérée qui provient de l'écroulement des blocs de glace issus de la chute de séracs. Pour le scénario de la figure 9 , à partir de l'année 2035 environ, le front de la partie supérieure du glacier ayant reculé, le flux du glacier n'alimenterait plus la chute de séracs. La partie inférieure ne bénéficierait alors plus d'alimentation par les chutes de séracs et serait alors uniquement constituée de glace stagnante qui fondra sur place. À partir des années 2040 environ, les projections indiquent que le front $\mathrm{du}$ glacier (partie supérieure) devrait laisser place à un lac qui va s'agrandir progressivement au cours des deux décennies suivantes. Ce lac se développera à l'endroit d'une profonde dépression (zone de surcreusement) du lit rocheux, en amont du verrou rocheux au niveau duquel se trouve l'actuelle chute de séracs de Lognan. La cartographie du lit rocheux sous le glacier par radar montre que le lac devrait atteindre un volume d'environ 12 millions de $\mathrm{m}^{3}$. Une fois que le lac apparaîtra, le vêlage (chute de séracs dans le lac, formant des icebergs) du glacier dans le lac devrait probablement entraîner un retrait plus rapide du front. Par ailleurs, des événements de vêlage de grande ampleur pourraient engendrer une vague susceptible de déborder du verrou rocheux avec un potentiel risque de crues dans la vallée. Après 2060, environ, cette projection indique que les affluents (glaciers des Améthystes, du Tour Noir et des Rognons) devraient être totalement déglacés. Néanmoins, nous n'avons pas de mesure directe des épaisseurs de ces affluents, ce qui accrôt les incertitudes sur leurs vitesses de désenglacement. Enfin, vers 2100, le glacier d'Argentière aurait, suivant ce scénario, quasiment disparu. Suivant le scénario le plus pessimiste (RCP 8.5), il est projeté que le glacier d'Argentière disparaisse avant 2080. Quel que soit le scénario envisagé, c'est la partie la plus épaisse (350 m environ), située aujourd'hui entre 2800 et $2900 \mathrm{~m}$ d'altitude qui disparaîtrait en dernier et non les zones les plus hautes, nettement moins épaisses.

S'agissant de la Mer de Glace, de plus grande superficie que le glacier d'Argentière et dont la zone d'accumulation est plus élevée, les projections indiquent qu'elle résisterait mieux quels que soient le modèle et les

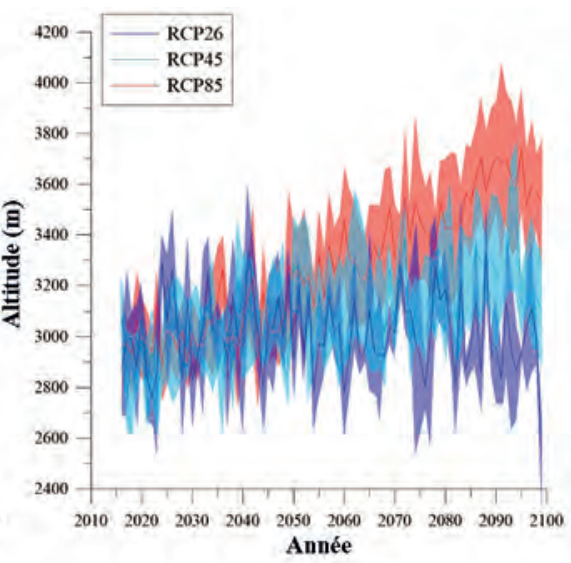

Figure 8. Évolution de la ligne d'équilibre annuelle (altitude en $\mathrm{m}$ ) calculée selon les trois scénarios et tous les modèles climatiques utilisés. Sont reportées les moyennes annuelles de tous les modèles climatiques pour chacun des scénarios et les écarts types associés.

scénarios envisagés. La Mer de Glace présente, comme Argentière, une chute de séracs (nommée chute de séracs du Géant). Les épaisseurs de glace dans cette chute de séracs sont probablement inférieures à $50 \mathrm{~m}$ (Lliboutry et Reynaud, 1981). Dans les prochaines décennies, il est projeté que le glacier de la Mer de Glace se scinde en deux, laissant une zone de glace stagnante à l'aval de cette rupture de pente. Étant donné les épaisseurs très élevées de la partie du glacier située à l'aval de la chute de séracs (nommée glacier du Tacul), d'environ $350 \mathrm{~m}$ aujourd'hui (Vincent et al., 2014), la fonte de cette glace devrait nécessiter plusieurs décennies, tandis que le front du glacier va continuer à reculer vers des altitudes plus élevées. Suivant le modèle CC-4.5, en 2100, le front du glacier aurait reculé de $7,2 \mathrm{~km}$ par rapport à sa position de 2018 (figure 10). Néanmoins, dans les parties les plus hautes, au pied de l'Aiguille du Midi, il resterait près de $150 \mathrm{~m}$ d'épaisseur de glace au col du Midi en 2100 (contre $200 \mathrm{~m}$ aujourd'hui). L'altitude de la surface du col du Midi serait par conséquent de $3450 \mathrm{~m}$ environ. Le glacier restant serait donc encore alimenté par une petite zone d'accumulation, car la ligne d'équilibre se situerait, elle, vers $3150 \mathrm{~m}$ (moyenne décennale pour la fin du siècle). En revanche, suivant le scénario RCP 8.5 et le modèle le plus pessimiste, la Mer de Glace devrait quasiment disparaître entre 2090 et 2100. Suivant ce scénario, la ligne

3. À la ligne d'équilibre, les bilans de masse de surface annuels sont nuls. Autrement dit, en ce lieu, l'ablation compense exactement l'accumulation. 
d'équilibre se situerait vers $3500 \mathrm{~m}$ au cours de la dernière décennie de ce siècle (figure 8). Dans cette hypothèse, excepté les plus hautes pentes du Mont-Blanc du Tacul, la zone d'accumulation serait réduite à néant.
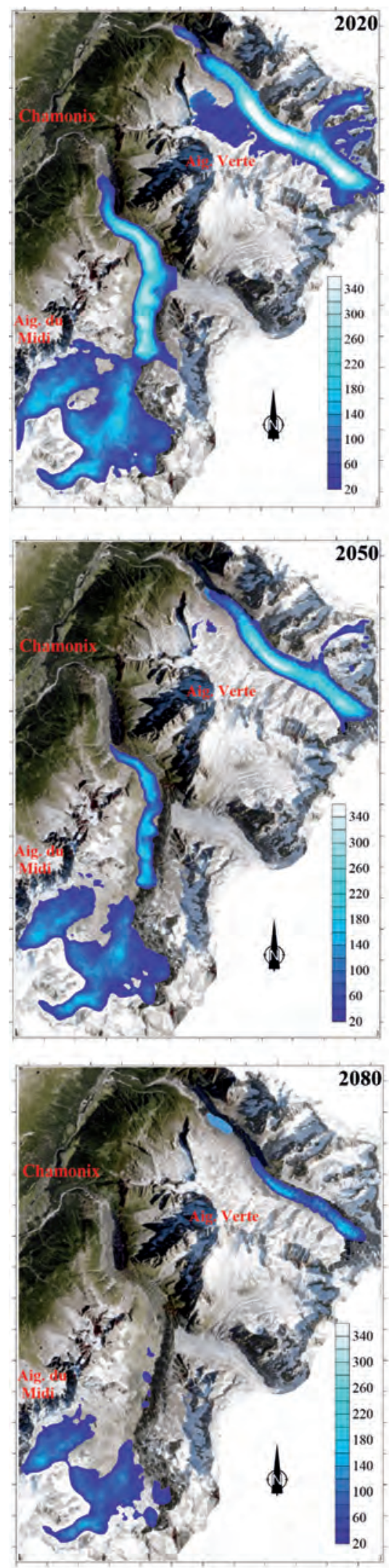

Enfin, on peut noter que, même avec des conditions climatiques et des bilans de masse de surface similaires à ceux des trois dernières décennies (1990-2018), ce qui n'est pas envisagé par les modèles climatiques, ces glaciers reculeront significativement, car ils ne sont pas en équilibre avec le climat des dernières décennies (Le Meur et al., 2007 ; Vincent et al., 2014).
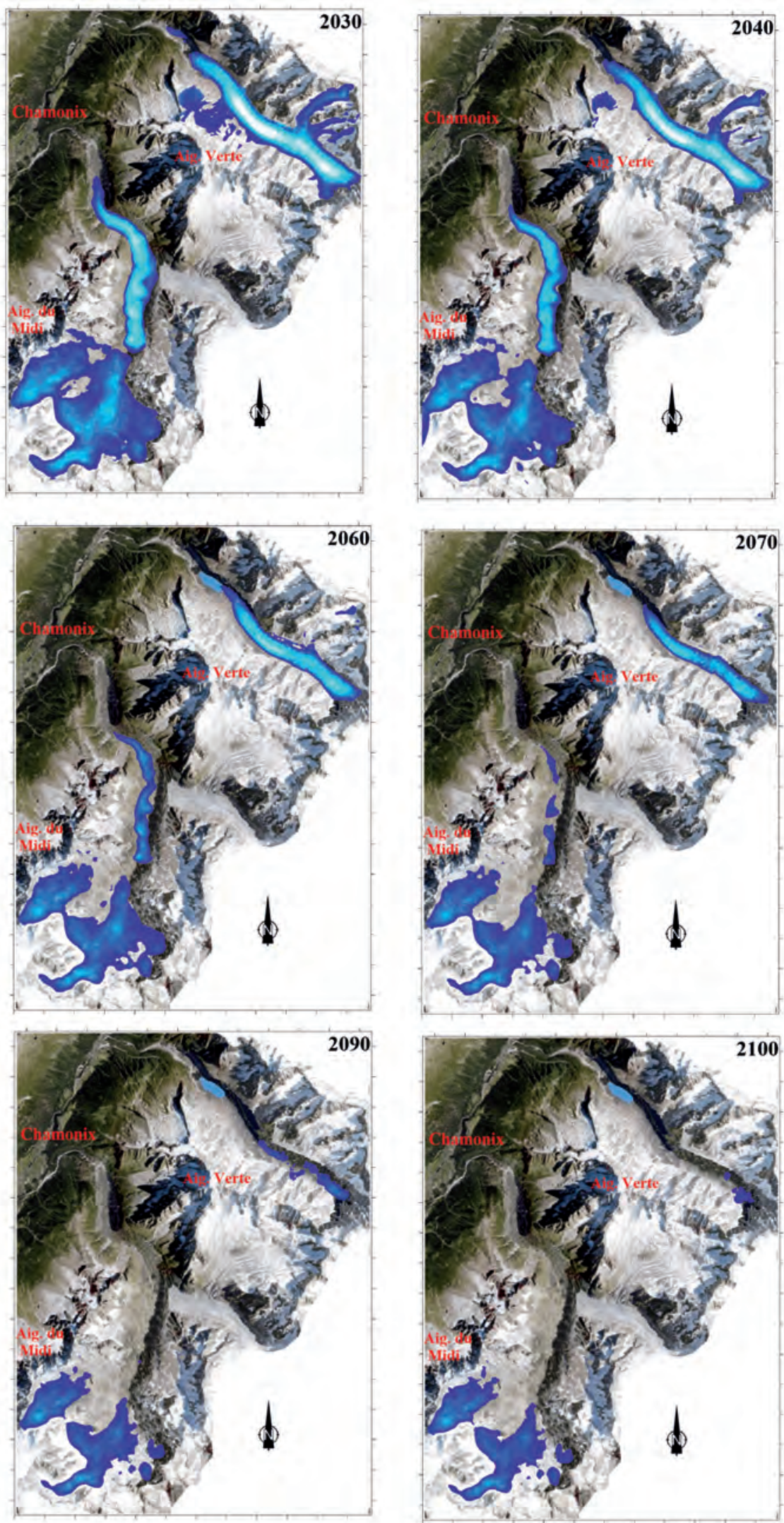

Figure 9. Extensions et épaisseurs (échelle de couleurs, en m) des glaciers d'Argentière et de la Mer de Glace simulées entre 2020 et 2100 sous le scénario climatique « modéré » RCP 4.5. L'échelle de couleur représente les épaisseurs de glace. 

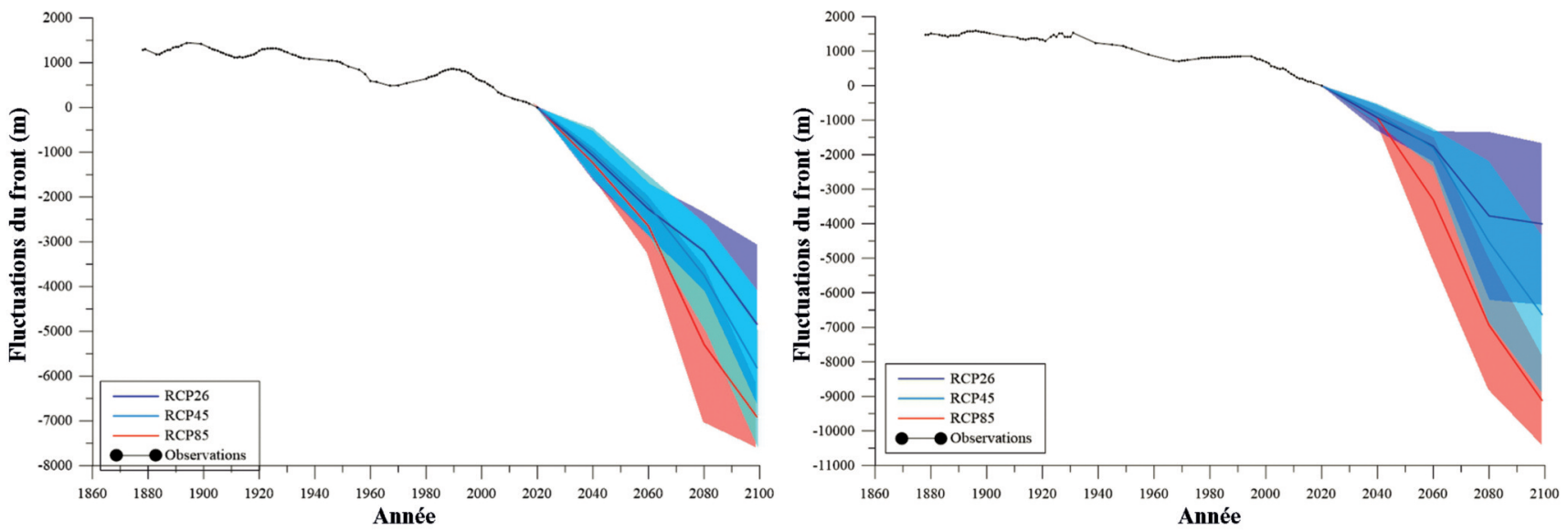

Figure 10. Fluctuations des fronts du glacier d'Argentière (en haut) de la Mer de Glace (en bas) dans le passé et dans le futur, pour les trois scénarios considérés et l'ensemble des simulations climatiques disponibles utilisées. La variation est exprimée en mètres par rapport à la position actuelle. Les points en noir représentent les observations depuis la fin du XIXe siècle jusqu'en 2017.

\section{Les incertitudes}

Les incertitudes de ces estimations sont liées (i) aux scénarios climatiques, (ii) aux modèles climatiques, (iii) à la connaissance du lit rocheux, (iv) au modèle qui détermine les bilans de masse et (v) à la méthode de modélisation utilisée pour calculer les variations d'épaisseur et de longueur.

Les scénarios et modèles climatiques fournissent une large fourchette de l'évolution des conditions. Ils dépendent essentiellement du scénario climatique considéré (RCP), qui dépend notamment des émissions de gaz à effet de serre à l'échelle de l'humanité (Stocker et al., 2013). L'étude fournit des résultats pour un ensemble de projections climatiques, correspondant à l'état de l'art en la matière (Verfaillie et al., 2018 ; Evin et al., 2019).

Le lit rocheux est très bien connu sous le glacier d'Argentière, excepté pour ses affluents. Dans ce cas, l'incertitude sur la connaissance du lit rocheux est faible au regard des autres incertitudes. La topographie du lit rocheux sous la Mer de Glace est bien connue dans la partie basse, à l'aval de la chute de séracs du Géant. En revanche, elle est mal connue dans la partie haute. Il en résulte nécessairement de plus fortes incertitudes sur les simulations après 2060 ou 2070, lorsque le front du glacier se situera dans cette zone haute. Si l'on considère que la variation d'épaisseur au front du glacier est de -7 mètres/an (moyenne simulée sur le $\mathrm{XXI}^{\mathrm{e}}$ siècle pour le modèle $\mathrm{CC}-4.5$ ), une erreur de $100 \mathrm{~m}$ sur l'épaisseur de glace considérée pourrait conduire à un biais temporel d'une quinzaine d'années environ dans nos résultats de simulation.
Le modèle degré-jour qui calcule les bilans de surface comporte également de fortes incertitudes, à travers les facteurs degré-jour qui peuvent varier à la fois dans l'espace et le temps. Néanmoins, Réveillet et al. (2017) ont montré que ces incertitudes sont probablement du second ordre par rapport à l'incertitude sur la précipitation neigeuse et sa rétroaction sur l'albédo de surface. En outre, à partir des incertitudes sur l'évolution des facteurs degré-jour dans le futur, estimée à $30 \%$ au maximum, et celles liées aux projections climatiques, on peut affirmer que l'incertitude sur les bilans de masse simulés à la fin du $\mathrm{XXI}^{\mathrm{e}}$ siècle liée aux paramètres du modèle de bilans de masse est bien inférieure à l'incertitude inhérente aux projections climatiques. Enfin, le modèle paramétré est un modèle empirique qui s'appuie sur des fonctions normalisées obtenues à partir des observations du passé. Ces fonctions pourraient changer dans le temps. On peut noter aussi que la fonction normalisée obtenue pour un glacier en crue peut être très différente de celle obtenue pour un glacier en décrue.
Même si les périodes de crue, dans l'avenir, sont peu probables, il peut en résulter une incertitude. Notons malgré tout que la conservation de la masse est assurée dans ce type de modèle. Aussi, les incertitudes ne concernent que la redistribution des variations d'épaisseur de glace suivant l'altitude. Pour évaluer ces incertitudes, les variations d'épaisseur normalisées (figure 6d) ont été systématiquement biaisées de $\pm 0,1$ pour la Mer de Glace. Les résultats indiquent des différences faibles entre ces simulations en comparaison des écarts entre les différents modèles climatiques et les différents scénarios considérés (non reportées sur les figures).

Enfin, les résultats du modèle paramétré ont été comparés avec les résultats d'une modélisation numérique en utilisant le modèle d'écoulement glaciaire Elmer/Ice (Gagliardini et al., 2013) sur le glacier d'Argentière pour lequel le lit rocheux est bien connu. Pour cette comparaison, nous avons utilisé les mêmes bilans de masse de surface calculés avec la même base de données climatiques (CC-4.5),

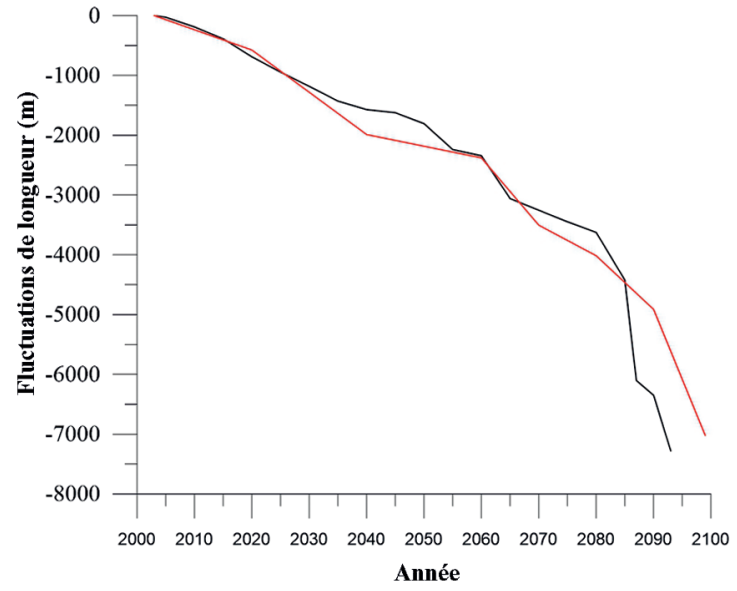

Figure 11. Fluctuations de longueur (en m) du glacier d'Argentière simulées entre 2003 et 2100 avec un couple GCM/RCM en RCP 4.5 (CC4.5 ) et le modèle Elmer/lce (courbe noire) ou la méthode paramétrée utilisée pour cette étude (courbe rouge) 
ce qui a permis d'évaluer les résultats du modèle paramétré d'une manière indépendante. Les évolutions de longueurs simulées avec le modèle Elmer/Ice et le modèle paramétré sont similaires (figure 11). Bien que les deux modèles soient contraints par les mêmes conditions de surface (bilans de surface) et assurent la conservation de la masse, la répartition des changements d'épaisseur est simulée d'une manière totalement différente dans les deux approches, en prenant en compte explicitement l'écoulement de la glace dans l'approche Elmer/Ice et en utilisant une fonction «normalisée » dans l'approche paramétrée. On peut en conclure que l'approche paramétrée est suffisante pour simuler les variations de surface et de volume dans le futur. Huss et al. (2010) avaient également montré, à partir d'une étude sur deux glaciers suisses, que les résultats entre les deux types de modèle étaient très similaires à cette échelle de temps. Les formations de chutes de séracs ou de lacs proglaciaires à venir, comme le lac d'Argentière, influenceront très probablement les fluctuations glaciaires dans le futur, mais les impacts concerneront des périodes de temps de quelques années, voire d'une ou deux décennies. Ainsi, avec la condition que la topographie du lit rocheux soit bien déterminée, les principales incertitudes proviennent des scénarios climatiques et des modèles climatiques.

\section{Conclusions}

Les simulations réalisées sur la Mer de Glace et le glacier d'Argentière indiquent que, quel que soit le scénario climatique envisagé, leurs volumes et leurs longueurs vont diminuer au cours du $\mathrm{XXI}^{\mathrm{e}}$ siècle. Les lignes d'équilibre pourraient atteindre des altitudes de 3150 et $3500 \mathrm{~m}$ au cours de la dernière décennie du $\mathrm{XXI}^{\mathrm{e}}$ siècle, pour les scénarios RCP 4.5 et RCP 8.5 respectivement. En comparaison, les altitudes des lignes d'équilibre de ces deux glaciers étaient situées vers 2900 $\mathrm{m}$ au cours des quatre dernières décennies

Avec un scénario RCP 4.5, les simulations indiquent que le glacier d'Argentière devrait disparaître vers la fin du XXI $\mathrm{XI}^{\mathrm{e}}$ siècle. Dans l'hypothèse la plus pessimiste (RCP 8.5), il pourrait disparaître avant 2080. À partir des années 2040 environ, le front du glacier va laisser place à un grand lac proglaciaire qui atteindra, au bout de deux décennies, un volume d'environ 12 millions de $\mathrm{m}^{3}$. Les résultats sur la Mer de Glace après 2060 sont beaucoup plus incertains, car la topographie du lit rocheux dans la partie supérieure du glacier est mal connue. Suivant le scénario RCP 4.5, le glacier ne disparaîtrait pas totalement et le front du glacier devrait reculer de $7,2 \mathrm{~km}$ entre 2018 et 2100 . Son épaisseur maximale au col du Midi ne dépasserait pas $150 \mathrm{~m}$. Suivant ce scénario, la Mer de Glace perdrait $80 \%$ de sa surface environ par rapport aux conditions du début du $\mathrm{XXI}^{\mathrm{e}}$ siècle. Suivant le scénario le plus pessimiste et le modèle le plus pessimiste, la Mer de Glace pourrait disparaître entre 2090 et 2100.

Compte tenu de notre bonne connaissance des épaisseurs de glace sur la grande partie de ces deux glaciers, les principales incertitudes de ces simulations sont liées aux incertitudes des modèles climatiques et aux différents scénarios climatiques.

Nos simulations sont comparables aux modélisations réalisées sur l'ensemble des glaciers des Alpes européennes (Huss, 2012) qui projettent, suivant différents scénarios, une diminution de 82 à $96 \%$ de la surface glaciaire d'ici 2100 .

\section{Remerciements}

Cette étude a bénéficié du soutien de l'Institut national des sciences de l'univers (Insu) du CNRS dans le cadre du service d'observation Glacioclim (les glaciers un observatoire du climat) (https://glacioclim.osug.fr). Cette étude a été rendue possible grâce aux financements et données issus du projet ANR VIP_Mont-Blanc (ANR-14-CE030006-03) et du LabEx OSUG@2020 (investissements d'avenir ANR10 LABX56). Le MNT LiDAR du glacier d'Argentière a été acquis dans le cadre du projet European Facility for Airborne Research Transnational Access (EUFAR TA, EU FP7 Integrating Activity) - project ISOTHERM (Ice, SnOw vegeTation HypERspectral Measurements). Cette étude utilise des résultats produits dans le cadre du projet GICC Adamont financé par le MTES et l'Onerc de 2015 à 2018, ainsi que dans le cadre du projet CDP Trajectories (IDEX Université Grenoble-Alpes) et Interreg OPCC2 Clim'Py. Deborah Verfaillie a été financée par le projet européen EUCP (H2020-SC5-2016-776613). L'IGE et le Centre d'étude de la neige du CNRM font partie du LabEX OSUG@2020. Étienne Berthier remercie le Cnes (Tosca) pour son soutien.

\section{Bibliographie}

Berthier E., Vincent C., 2012. Relative contribution of surface mass balance and ice flux changes to the accelerated thinning of the Mer de Glace (Alps) over $1979-2008$. J. Glaciol., 58, 501-512. doi: 10.3189/2012JoG11J083

Berthier E.. et al., 2014. Glacier topography and elevation changes derived from Pléiades sub-meter stereo images. The Cryosphere, 8, 2275-2291. doi: 10.5194/tc-8-22752014

Brondex J., Gagliardini 0., Gillet-Chaulet F., Durand G., 2017. Sensitivity of grounding line dynamics to the choice of the friction law. J. Glaciol., 63, 854-866. doi: 10.1017/jog.2017.51

Durand Y., Laternser M., Giraud G., Etchevers P., Lesaffre B., Merindol L., 2009. Reanalysis of 44 yr of climate in the French Alps (1958-2002): Methodology, model validation, climatology, and trends for air temperature and precipitation. J. Appl. Meteorol. Climatol., 48, 429-449. doi: 10.1175/2008JAMC1808.1

Evin G., Hingray B., Blanchet J., Eckert N., Morin S., Verfaillie D., 2019. Partitioning uncertainty components of an incomplete ensemble of climate projections using data augmentation. J. Climate. doi: 10.1175/JCLI-D-18-0606.1

Farinotti D., Brinkerhoff D.J., Clarke G.K.C., Fürst J.J., Frey H., Gantayat P., Gillet-Chaulet F. et al., 2017. How accurate are estimates of glacier ice thickness? Results from ITMIX, the Ice Thickness Models Intercomparison eXperiment. The Cryosphere, 11, 949-970. doi: 10.5194/tc-11-949-2017

Fischer M., Huss M., Hoelzle M., 2015. Surface elevation and mass changes of all Swiss glaciers 1980-2010. The Cryosphere, 9, 525-540. doi: 10.5194/tc-9-525-2015

Gabbi J., Carenzo M., Pellicciotti F., Bauder A., Funk M., 2014. A comparison of empirical and physically based glacier surface melt models for long-term simulations of glacier response. J. Glaciol., 60, 1140-1154. doi: 10.3189/2014JoG14J011 
Gagliardini 0., Zwinger T., Gillet-Chaulet F., Durand G., Favier L., Fleurian B. de, Greve R. et al., 2013. Capabilities and performance of Elmer/lce, a new-generation ice sheet model. Geosci. Model Dev., 6, 1299-1318. doi: 10.5194/gmd-6-1299-2013

Gilbert A., Leinss S., Kargel J., Kääb A., Gascoin S., Leonard G., Berthier E. et al., 2018. Mechanisms leading to the 2016 giant twin glacier collapses, Aru Range, Tibet. The Cryosphere, 12, 2883-2900. doi: 10.5194/tc-12-2883-2018

Huss M., 2011. Present and future contribution of glacier storage change to runoff from macroscale drainage basins in Europe. Water Resour. Res., 47, W07511. doi: 10.1029/2010WR010299

Huss M., 2012. Extrapolating glacier mass balance to the mountain-range scale: the European Alps 1900-2100. The Cryosphere, 6, 713-727. doi: 10.5194/tc-6-713-2012

Huss M., Farinotti D., 2012. Distributed ice thickness and volume of all glaciers around the globe. J. Geophys. Res. Earth Surf., 117, F04010. doi: 10.1029/2012JF002523

Huss M., Hock R., 2018. Global-scale hydrological response to future glacier mass loss. Nat. Clim. Change, 8, 135-140. doi: 10.1038/s41558-017-0049-x

Huss M., Jouvet G., Farinotti D., Bauder A., 2010. Future high-mountain hydrology: a new parameterization of glacier retreat. Hydrol. Earth Syst. Sci., 14, 815-829. doi: 10.5194/hess-14-815-2010

Huss M., Bookhagen B., Huggel C., Jacobsen D., Bradley R.S., Clague J.J., Vuille M. et al., 2017. Toward mountains without permanent snow and ice. Earth's Future, 5, 418435. doi: 10.1002/2016EF000514

Jouvet G., Huss M., Funk M., Blatter H., 2011. Modelling the retreat of Grosser Aletschgletscher, Switzerland, in a changing climate. J. Glaciol., 57, 1033-1045. doi: $10.3189 / 002214311798843359$

Kaser G., Großhauser M., Marzeion B., 2010. Contribution potential of glaciers to water availability in different climate regimes. Proc. Natl Acad. Sci., 107, $20223-20227$. doi: 10.1073/pnas.1008162107

Le Meur E., Gerbaux M., Schafer M., Vincent C., 2007. Disappearance of an Alpine glacier over the 21st century simulated from modeling its future surface mass balance. Earth Planet. Sci. Lett., 261, 367-374. doi: 10.1016/j.epsl.2007.07.022

Lliboutry L., Reynaud L., 1981. 'Global dynamics' of a temperate valley glacier, Mer de Glace, and past velocities deduced from Forbes bands. J. Glaciol., 27, 207-226.

Oerlemans J. (Ed.), 2001. Glaciers and climate change. Rotterdam, A.A. Balkema Publishers.

Rabatel A., Sanchez O., Vincent C., Six D., 2018. Estimation of glacier thickness from surface mass balance and ice flow velocities: A case study on Argentière Glacier, France. Front. Earth Sci., 6, 112. doi: 10.3389/feart.2018.00112

Réveillet M., Vincent C., Six D., Rabatel A., 2017. Which empirical model is best suited to simulate glacier mass balances? J. Glaciol., 63, 39-54. doi: 10.1017/jog.2016.110

Réveillet M., Six D., Vincent C., Rabatel A., Dumont M., Lafaysse M., Morin S. et al., 2018. Relative performance of empirical and physical models in assessing the seasonal and annual glacier surface mass balance of Saint-Sorlin Glacier (French Alps). The Cryosphere, 12, 1367-1386. doi: 10.5194/tc-12-1367-2018

Stocker T.F., Qin D., Plattner G.K., Tignor M., Allen S.K., Boschung J., Nauels A. et al., 2013. IPCC, 2013: Climate Change 2013: The Physical Science Basis. Contribution of Working Group I to the Fifth Assessment Report of the Intergovernmental Panel on Climate Change. Cambridge, United Kingdom and New York, NY, USA: Cambridge University Press.

Thibert E., Sielenou P.D., Vionnet V., Eckert N., Vincent C., 2018. Causes of glacier melt extremes in the Alps since 1949. Geophys. Res. Lett., 45, 817-825. doi: 10.1002/2017GL076333

Vallot J., 1905. Annales de l'Observatoire du Mont-Blanc. Tome I à VI. Paris, G. Steinheil.

Verfaillie D., Deque M., Morin S., Lafaysse M., 2017. The method ADAMONT v1.0 for statistical adjustment of climate projections applicable to energy balance land surface models. Geosci. Model Dev., 10, 4257-4283. doi: 10.5194/gmd-10-4257-2017

Verfaillie D., Lafaysse M., Deque M., Eckert N., Lejeune Y., Morin S., 2018. Multi-component ensembles of future meteorological and natural snow conditions for $1500 \mathrm{~m}$ altitude in the Chartreuse mountain range, Northern French Alps. The Cryosphere, 12, 1249-1271. doi: 10.5194/tc-12-1249-2018

Vincent C., 2002. Influence of climate change over the 20th century on four French glacier mass balances. J. Geophys. Res. - Atmos., 107, D19, 4375, doi: 10.1029/2001JD000832

Vincent C., Moreau L., 2016. Sliding velocity fluctuations and subglacial hydrology over the last two decades on Argentiere glacier, Mont Blanc area. J. Glaciol., 62, 805815. doi: 10.1017/jog.2016.35

Vincent C., Soruco A., Six D., Le Meur E., 2009. Glacier thickening and decay analysis from 50 years of glaciological observations performed on Glacier d'Argentière, Mont Blanc area, France. Ann. Glaciol., 50, 73-79. doi: 10.3189/172756409787769500

Vincent C., Harter M., Gilbert A., Berthier E., Six D., 2014. Future fluctuations of Mer de Glace, French Alps, assessed using a parameterized model calibrated with past thickness changes. Ann. Glaciol., 55, 15-24. doi: 10.3189/2014AoG66A050

Vincent C., Fischer A., Mayer C., Bauder A., Galos S.P., Funk M., Thibert E. et al., 2017. Common climatic signal from glaciers in the European Alps over the last 50 years. Geophys. Res. Lett., 44, 1376-1383. doi: 10.1002/2016GL072094

Zemp M., Thibert E., Huss M., Stumm D., Rolstad Denby C., Nuth C., Nussbaumer S. U. et al., 2013. Reanalysing glacier mass balance measurement series. The Cryosphere, 7, 1227-1245. doi: 10.5194/tc-7-1227-2013

Zemp M., Frey H., Gaertner-Roer I., Nussbaumer S.U., Hoelzle M., Paul F., Haeberli W. et al., 2015. Historically unprecedented global glacier decline in the early 21st century. J. Glaciol., 61, 745-762. doi: 10.3189/2015JoG15J017

Zemp M., Huss M., Thibert E., Eckert N., McNabb R., Huber J., Barandun M. et al., 2019. Global glacier mass balances and their contributions to sea-level rise from 1961 to 2016. Nature, 568, 382-386, doi: 10.1038/s41586-019-1071-0 